\title{
Pensar la materialidad de los afectos con Giordano Bruno ${ }^{1}$
}

\author{
Valentina Bulo
}

Resumen: Este ensayo se propone hacer una lectura del texto "De los vínculos en general" de Giordano Bruno a partir de su propia reelaboración de la idea de materia y en vistas al planteamiento actual de la afectividad. No buscamos hacer una lectura historiográfica de Bruno sino más bien nos esforzamos en traer su filosofía para replantearla desde nuestras actuales problemáticas. Para esto partiremos describiendo algunos rasgos relevantes de la idea de materia en Giordano Bruno para luego desde allí acercarnos a su teoría general de los vínculos. Finalmente realizaremos algunas consideraciones de la afectividad a partir de los vínculos brunianos.

Palavras-chave: Giordano Bruno; afectividad; vínculos; materia.

Abstract: This essay proposes a reading of Giordano Bruno's "A General Account of Bonding", starting from his own re-elaboration of the idea of matter in light of the current approach of emotion. We do not intend to carry out a reading of Bruno from the historiographical point of view, but to strive to bring his philosophy in order to reformulate it according to our current predicament. So as to achieve this goal, we will begin describing some relevant features of the idea of matter in Giordano Bruno in order to be able to attain a closer view of his general theory of bonding. Finally, we will analyze some considerations of affectivity starting from Brunian bondings.

Keywords: Giordano Bruno; affectivity; bondings; matter.

\section{A Humberto Giannini}

...en la cabeza nieve, en la frente surcos, en la nariz gafas, en el mentón harina, en las manos bastón, en los pies plomo; en la cabeza -digo- la fantasía recta, la reflexión solícita, la memoria retentiva; en la frente la aprehensión sensata, en los ojos la prudencia, en la nariz la sagacidad, en las orejas la atención, en la lengua la verdad, en el pecho la sinceridad, en el corazón los sentimientos ordenados, en los hombros la paciencia, en la espalda el olvido de las ofensas, en el estómago la discreción, en el vientre la sobriedad, en el pecho la continencia, en las piernas la constancia, en los pies la rectitud... (BRUNO, 1995, p.113)

1 Este escrito forma parte del Proyecto de Investigación Fondecyt Regular n¹150266 del que soy Investigadora Responsable. 
Pensar la afectividad desde una materialidad parece en principio un contrasentido, al menos si intentamos "rescatar" el orden de los afectos de sus articulaciones con los estratos "inferiores del cuerpo" para desplazarlos lo más posible del lado de las "funciones superiores del alma". Relacionar los afectos con la materia sería un ejercicio que vuelve a encasillar al hombre, en tanto seres afectivos con "lo más bajo". A menos que reconvirtamos también la idea de materia y ampliemos de tal modo la idea de afectividad que en ella quepan no sólo los afectos humanos sino de todas las cosas del mundo y además profundicemos con tal radicalidad esta afectividad que ella involucre también los cambios físicos, las pasiones-acciones o el curso de los átomos. Pensamos que este trabajo ha sido elaborado en muchos aspectos por Giordano Bruno e intentaremos dar cuenta de algunos de ellos. Para esto partiremos demarcando algunos rasgos relevantes de la idea de materia en Giordano Bruno para luego desde allí acercarnos a su teoría general de los vínculos. Finalmente realizaremos algunas consideraciones de la afectividad a partir de los vínculos brunianos.

\section{El mundo como materia animada que se explica}

La materia en la filosofía del Nolano no es en absoluto una mera carencia, negación o vacío, sino que es un vacío lleno de todo lo posible, entendiendo posibilidad como el poseer en "acto" todas las formas haciéndolas surgir desde sí misma. "La materia, por ser en acto todo cuanto puede ser, tiene todas las medidas, todas las especies de figuras y dimensiones" (BRUNO, 1941, p.122) ¿Cómo se puede ser en acto todo lo posible? Siéndolo todo, la materia es aquello desde donde emerge toda forma, y este "desde donde" no es algo exterior a las formas, sino las formas mismas en su "complicación" (Cf. BRUNO, 1941, p.133ss, 117). Se trata, pues, de una lectura de la positividad de la materia con aquellos caracteres que han sido trabajados predominantemente (especialmente en Aristóteles) de forma negativa.

La materia, siguiendo esta línea de lectura, es comprendedora e implicadora de todo, no como una potencia que "puede ser todo" sino el físico germen de las formas que es interior a ellas como una implicación actual. Por eso Bruno le reprocha a Aristóteles el considerar de un modo más restringido el acto reduciéndolo a la mera explicación de la forma, "Aristóteles le llamó al acto más bien a la explicación de la forma que a la implicación" (BRUNO, 1941, p.128); a la materia sólo le quedaría ser una potencia a la que le falta determinación, en cambio en Bruno la materia "no es ese propte nihil, esa potencia pura, desnuda, sin acto, ni virtud ni perfección...la digo privada de forma y sin ellas, no como el hielo está privado del calor y como el abismo de luz, sino como la mujer preñada está sin su prole, que ella produce y saca de sí misma; y como la tierra, sin luz durante la noche en nuestro hemisferio, que, con su movimiento, es capaz de recobrar" (BRUNO, 1941, p.126). Se trata de la materia entendida como positividad y sobredeterminación. 
Si pensamos el ejemplo de la maternidad parece obvio que la madre es el "desde donde" proviene el hijo, pero aquí el "estar sin" su prole, siendo que ya es madre, casi podríamos decir que es un modo de "estar con". El "estar sin" se refiere obviamente al hecho de que su prole aún no ha nacido, no se ha presentado al mundo como algo distinto o exterior a ella; la madre ya es madre y su hijo también, son sus existencias las que están complicadas, el hijo es interior a la madre no sólo porque está "en" su vientre así como un libro está "en" la mesa sino que son de alguna manera lo mismo. Es justo la complicación de las formas. La materia es madre materia pues "desde ella" y "en ella" todas las formas son complicadamente, plegadamente, cada una de sus partes.

Este es el sentido también en que el infinito universo contiene infinitos mundos, los cuales poseen cada uno un principio interior de movimiento, que es su alma. "... Estos cuerpos mundiales se mueven en la región etérea y no están más fijos ni clavados en cuerpo alguno de lo que está la tierra, que es uno de estos cuerpos; por ello podemos probar de ésta que, por el interior animal ínsito, circunda el propio centro y el sol, de varias maneras" (GÓMEZ, 1973, p.180). De este modo podemos ver que para Bruno la tierra, al poseer este "animal" en sí, se mueve en torno de sí misma recobrando y perdiendo luz y oscuridad respecto del sol en sus hemisferios. Es la tierra que desde sí misma da luz y oscuridad a sus hemisferios, así como la materia complica y explica las formas, pero no como potencia en la indeterminación, oscuridad y complicación de las formas, y acto en lo determinado, explicado, la luz; sino que la indeterminación, la complicación de las formas es un "estado" actual de la materia, quien "desde sí" irá rotando para que estas formas se vayan explicando, iluminando y a su vez otras se compliquen.

Si la materia es el principio desde donde y en donde todo emerge, si la materia son las cosas mismas en su "complicación", es fácil imaginar que la explicación de las cosas estará dada para Giordano por un principio formal, quien está a cargo, por decirlo de algún modo, de la determinación de cada cosa, de su presencia en el mundo como "tal". Este principio formal no es un concepto de razón según la cual unas cosas sean distintas de las otras, sino que es un principio vital, que anima a todo lo existente en el mundo, por esta razón, absolutamente todo posee un alma, no sólo árboles y hombre, sino piedras y átomos, "el espíritu se halla en todas las cosas, y no hay corpúsculo, por mínimo que sea, que no contenga en sí mismo algo que no esté animado" (BRUNO, 1941, p.73s).

La animación de cada uno de los elementos del mundo está fundada en una unidad de éstos, el mundo mismo posee un alma y por eso ella se encuentra en cada corpúsculo de él, no una "parte" de la Gran Alma, sino toda ella en cada cosa existente, nuestro Nolano habla expresamente del Alma del Mundo, vivificación del universo, unificación a la vez de cada una de las cosas en él existentes, verdadera Alma- entendimiento del mundo (GÓMEZ, 1973, p.66s). Recordemos que Bruno concibe un universo contenedor de infinitos mundos y el alma del mundo lo es de todos ellos, es una verdadera "alma del infinito", pues nada queda fuera de ella, todo lo que es está animado enteramente por esta alma. 
El alma del mundo es el principio formal constitutivo del universo y de lo que en él está contenido. Digo que si la vida se halla en todas las cosas, el alma viene a ser la forma de todas las cosas: por doquier ella preside en la materia y señorea en los compuestos; efectúa la composición y la consistencia de las partes (BRUNO, 1941, p.76).

Podríamos afirmar entonces que en Bruno hay una animificación de la materia a la vez que una materialización del alma. Esta unidad es tan radical que no hay exterioridad entre ellas, la una es completamente interior a la "otra". Esta misma unidad-identidad-diferencia acontece con acto-potencia, ser-ente, máximo-mínimo, etc. Y es que Bruno radicaliza la unidad de todo, del Todo, a tal nivel que todos los contrarios se unen, pues nada queda fuera de esta unidad, todo es uno, por lo tanto, uno son también los contrarios; Bruno lo afirma explícitamente más de una vez : "todas las cosas son uno, el cual tiene y comprende en sí mismo todas las cosas ; y puesto que en él están todas las formas, consiguientemente todas las definiciones les convienen $y$, por tanto, los enunciados contradictorios son verdaderos" (BRUNO, 1941, p.144ss y cf. 151s., 152, 156). Literalmente "todo está en todas partes", "todo es todo", en donde podríamos decir que, en cada parte está el todo y el todo es cada parte. Nada es exterior a ella, unidad circular en la que la parte sólo es en el todo y el todo es en la parte, o lo que es lo mismo, en cada parte está completamente contenido el todo y el todo es la parte misma. Bruno ocupa la imagen de circularidad para explicarlo, "el universo es todo él centro, o bien que la circunferencia del universo está por todas partes y en ninguna, pero el centro no es diferente de la circunferencia" (BRUNO, 1941, p.138).

Los términos "complicación" y "explicación", no han de ser entendidos únicamente, como lo hemos mostrado hasta ahora, en su sentido "ontológico", sino con un alcance más radical y es que el ente junto con ser una "explicación" del uno, en el sentido de determinar lo que estaba indeterminado, "distinguirse" y exteriorizarse de algún modo, tiene un real (no metafórico) sentido de explicación discursiva; las cosas son, para Bruno la explicación de Dios mismo. La divinidad se explica en las cosas, las cosas son la autoexplicación de lo divino. Bruno, al hablar del alma del mundo, la llama también "Entendimiento universal", como la facultad del alma del mundo (cf. BRUNO, 1941, p.64ss) además de afirmar que “Dios está en las cosas y la divinidad... se participa a sí misma, digo, el ser, la vida y el entendimiento" (BRUNO, 1995, p.209). La complicación sería entonces Dios mismo, la pura unidad, la cual se explica, se comprehende, se "realiza" en el discurso infinito del universo. "La inteligencia primera todo lo comprende en una idea perfectísima; la mente divina, la unidad absoluta [lo entiende] sin especie alguna: es a la vez lo que comprende y lo que es comprendido" (BRUNO, 1941, p.148).

Aquel que lee este discurso infinito del universo para encontrar allí la explicación de Dios será el hombre, que como todas las cosas es espejo del infinito y junto con eso 
es el lugar en donde lo divino se mira a sí mismo, como también viceversa, el hombre ve al universo en sí mismo, y ese sí mismo es todas las cosas. “¿Cuál es aquel ojo que ve las demás cosas como se ve a sí mismo? Aquel que en sí mismo ve todas las cosas y el mismo es todas las cosas..., pues así como... no podemos ver la semejanza del propio ojo sino en el espejo, así tampoco nuestro intelecto [ve] a sí mismo en sí mismo, si no en una cierta especie exterior, el simulacro, imagen, figura, signo" (GÓMEZ, 1973, p.336). De aquí nace el fundamento de toda magia y de la filosofía pues sólo porque en cada cosa está el todo es posible llegar a la unidad "a través" de las cosas, si todo está en todas partes hay una suerte de convertibilidad que posibilita los ritos mágicos.

Una es la divinidad que se encuentra en todas las cosas, la cual, difundiéndose y comunicándose de innumerables maneras, tiene nombres innumerables, con razones, propias y adecuadas a cada uno, se la busca, al tiempo que se la honra y se la adora con ritos innumerables, porque innumerables son las clases de gracia que de ella se impetran (BRUNO, 1995, p.262).

\section{Materialidad de los Vínculos; una lectura de la mano de Lucrecio}

Es importante destacar, ya que es desde donde leeremos "De vinculis", la enorme influencia que Bruno tiene de Lucrecio y Epicuro, tanto en su estilo, especialmente en sus diálogos italianos, como en el modo de presentarse a sí mismo, claramente moldeado por el modo de alabanzas que hace Lucrecio a Epicuro en el De rerum natura, "solo que los papeles que en Epicuro y Lucrecio aparecen separados y propios de personas diferentes (los papeles de liberador y divulgador) se dan en Bruno unidos, por lo que el pensador italiano es a la vez el rebelde liberador de la humanidad y el vocero divulgador de la buena nueva" (GRANADA, 2005, p.96s.).

La lectura que proponemos aquí, si bien reconoce que innegablemente el lenguaje de Bruno es aristotélico con claras marcas platonizantes, sus tesis pueden ser leídas también desde De rerum natura: como lo afirma Humberto Giannini en su texto de "Los heroicos furores" la materia en Bruno es equiparable a la naturaleza, y aquí agregamos, a la naturaleza de las cosas en el sentido lucreciano.

El omnicentrismo bruniano dialoga directamente con las tesis de Copérnico sobre la cuestión del centro del universo ${ }^{2}$, donde Bruno, además de negar el geocentrismo afirmará el centro para cada esfera, dada la infinitud del universo. Sin obviar lo anterior,

2 Aunque no nos adentraremos en este tema, es necesario al menos mencionar que la cuestión del "centro del mundo" tiene que ver directamente con el lugar de Dios, "Para Bruno, Dios es centro interior del mundo. Esto es lo primero. Pero, además, tal centro está en todas partes y en cualquiera (omnicentrismo). Por lo que no existe 'un lugar' privilegiado del cielo - un Empireo- reservado sólo a la divinidad... Ahora bien, puede mostrarse en diversos textos que Giordano Bruno no llega a identificar a Dios con la naturaleza o el mundo. El Artífice interno (il datore delle forme) es en cada momento, y no sucesivamente, todo el poder que tiene. En este sentido el Dios bruniano trasciende esa Naturaleza que anima, ilumina y dirige desde dentro. No cabría, pues, pensarlo como idéntico a su obra" (Giannini, 2000). 
pensamos que la afirmación "todo es centro", además de su sentido macrocósmico, también debe entenderse como reordenación de nuestro mundo: la naturaleza de las cosas apunta a un omnicentrismo donde el centro también son las especies de animales, los dioses, las piedras y una naturaleza que se constituye a partir de la inclinación (clinamen) de los átomos. Desde una materia así puede adquirir mayor sentido su idea de los vínculos para repensar la afectividad. Es lo que intentaremos mostrar ahora.

Los vínculos en Giordano Bruno son acoplamientos, una fusión química es un vínculo, un león que come a su presa establece un vínculo, el girasol que se mueve al compás del sol está vinculado a él, dos miradas que se encuentran por segundos, un enamoramiento, un instante de odio, un ejercicio de dominación. Si pensamos en esta infinidad de vínculos desde la concepción de materia expuesta antes, nos topamos con que no sólo "toda realidad es vinculable" (BRUNO, 2007, p.91) sino que "en cada especie yacen gérmenes de atracción por todas las demás" (BRUNO, 2007, p.95). Si todo está en todas partes cada uno de nosotros posee una especie de vinculatividad infinita. "En verdad toda la sustancia y consistencia y, para usar un término difícil, la hipóstasis de la realidad es una especie de gran vínculo" (BRUNO, 2007, p.108).

Contempla, en los seres vivientes, la amistad y la enemistad, la simpatía y la antipatía, la afinidad y la diferencia y las circunstancias de todas estas cosas; luego, afronta, de acuerdo a cierto orden y analogía, las realidades particulares e individuales de la especie humana tomadas singularmente.Entonces: en primer lugar las especies de los otros vivientes, una por una y luego todas juntas; finalmente, todas las otras especies de cosas. Entenderás de qué variedad y disponibilidad de vínculos tienes necesidad (BRUNO, 2007, p.91).

Giordano Bruno está diagramando una suerte de retícula ${ }^{3}$ de vínculos, una estructura invisible de la vinculatividad entre unas cosas y otras. Giordano no se cansa de afirmar el carácter plural de los vínculos; trabaja treinta "líneas de fuerza" (vinvientis intentiones) del campo vinculante, que son algo así como las fuerzas que concurren a la formación de vínculos, él las Ilama fuerzas que ligan y de ellas al menos seis aluden expresamente a su multiplicidad, variedad y diferencia.

Esta pluralidad no es una "falta de unidad" como una carencia sino que es tratada como una ventaja y el texto de Bruno convoca al lector a aumentar el ejercicio de la amplitud de vínculos. Del mismo modo que en La expulsión de la bestia triunfante Júpiter lamenta la reducción de los muchos dioses al dominio del monoteísmo (126), Bruno considerará en posición desventajada a aquellos que sólo son capaces de establecer pocos tipos de vínculos o de limitarse sólo a uno. Su estilo de escritura por acumulación, en donde va agregando casos, ejemplos, caracteres, modos, situaciones, muestra esta tendencia multiplicativa al infinito y su enseñanza de los vínculos estará siempre marcada con

3 Este término está usado en la traducción castellana de Gatto e Ires para aludir a universaliter (BRUNO 2007, p.70). 
el énfasis en la prioridad de las múltiples variaciones de cada caso de vínculo, justamente adquirir la sabiduría de ligar tiene que ver con ello.

Los vínculos son sutiles, aquello que es sujetado por vínculos apenas aflora a la sensibilidad desde sus profundidades: es posible examinarlo sólo fugazmente, como desde una superficie elevada, y se encuentra además sujeto a transformaciones momento a momento...por más que de hecho la materia sea abierta a innumerables formas, su forma actual no se encuentra a igual distancia de todas las otras posibles (BRUNO, 2007, p.83).

La multiplicidad de vínculos está determinada "según la multiplicidad de las partes de la materia" (BRUNO, 2007, p.72). Las fuerzas que ligan, además funcionan como una energía que "da forma a la materia en la variedad infinita de sus situaciones; vivifica, aplaca, acaricia y excita toda la realidad, y la ordena, la vitaliza, la gobierna y la inflama" (BRUNO, 2007, p.71).

A partir de una concepción omnicéntrica del mundo, las jerarquías, bien que en Bruno no desaparecen del todo, pierden su carácter determinante en el orden del universo, pues son los vínculos y la liberación de vínculos en donde se producen las variaciones de estados de las cosas y su movimiento. Los vínculos son los que gatillan la explicación del mundo, en el sentido descrito antes, y tal como lo trabaja Lucrecio en De rerum natura, la desviación del curso de los átomos, el clinamen (LUCRECIO, 1994, p.147) será la posibilitación del encuentro entre unos y otros.

De todos modos, como fondo de la pluralidad de vínculos, el amoroso cumplirá la función de ser el vínculo primordial y la base posibilitante de cualquier otro; es lo que ya Ficino llamara copula mundi (FICINO, 1986), se trata del amor como fondo desde el cual cualquier vínculo se realiza, pues él es el que provoca la inclinación necesaria para el encuentro de los cuerpos, si en Ficino el orden del amor distribuirá las partes del universo de un modo estrictamente jerárquico, en la cosmología bruniana la jerarquía tenderá a desaparecer (GONZÁLEZ, 1987, p.18).

En De rerum natura Venus aparece como la fuerza vivificadora de la naturaleza que introduce el deseo en los seres vivos y que hace nacer y crecer a todas las cosas, por lo que se la representa a través de la llegada de la primavera. Lucrecio identifica a Venus con voluptas, el placer dinámico de la sexualidad y la generación (cf. MOLINA, 1998, p.243). En Bruno, de un modo confluyente al de Lucrecio, "quien vincula, no encadena a sí el alma si no la ha arrebatado; no la arrebata si no encadenada; no la encadena si no se enlaza a ella; no se enlaza si no la alcanza; no la alcanza si no a través de un impetuoso acercamiento; no se acerca si no se inclina, más bien declina, hacia ella; no se inclina si no lo mueve el deseo, el apetito; no apetece si no ha madurado un conocimiento; pero no puede madurar un conocimiento si el objeto no se hace presente en figura o en simulacro ante sus ojos, oídos, o ante las percepciones del oído interno" (BRUNO, 2007, p.84). 
Parafraseando lo que afirma Michel Serres en su libro sobre Lucrecio, que hay en él una opción por una física de Venus y no de Marte (1994, p.14) en el sentido de una ordenación cósmica que parte de esa inclinación o desviación del curso regular de los ciclos a partir de un fondo de amor y voluptuosidad; nos atrevemos a afirmar aquí, que en Bruno la idea de los vínculos y del amor como vinculante primordial tiene en parte este sentido lucreciano ${ }^{4}$.

\section{Vínculos y afectividad}

Si bien esta teoría de los vínculos es de "amplitud cósmica" y la leemos como una suerte de conjunción de fuerzas que posibilitan y gatillan las relaciones entre unos cuerpos y otros, el texto de los vínculos de Giordano Bruno va dirigido a los hombres, y más específicamente a ellos en tanto establecen diferentes relaciones civiles:

Es posible imaginar un mismo argumento transmigrando de forma en forma, de figura en figura, de manera tal que para vincularlo se deban utilizar permanentemente nuevas especies de nudos cada vez. Añádase a esto la valoración de los modos de vida de los hombres, los cuales pueden ser jóvenes o viejos; en cuanto a la ubicación civil, pueden ser mediocres o nobles o ricos y poderosos y afortunados; supóngase además que son envidiosos y ambiciosos; o soldados y mercaderes y otros de esos tipos. Son estas las personas que asumen los diferentes roles de la administración civil, donde obran como medios o instrumentos, lo cual les pone la cuestión de vincularlos a sí. No parece, en definitiva, que realidad alguna escape a una reflexión sobre las relaciones civiles desde esta perspectiva: en la medida en que los hombres vinculan o están bajo vínculos o son ellos mismos o circunstancias vinculantes (BRUNO, 1997, p.70).

El objetivo de Giordano Bruno en el texto parece dirigirse a la regulación de las relaciones civiles y pensamos que en este sentido puede ser relacionado directamente con el objetivo de Aristóteles en el Libro II de la Retórica, -que Heidegger ha denominado "la primera hermenéutica sistemática de la cotidianeidad del convivir" (1997, p.163), que al tratar las pasiones las describe como modos de civilidad en donde unos apasionan a otros y los convencen, Aristóteles describe el modo de acontecer las diversas pasiones, sus causas y afinidades e incompatibilidades para la determinación en la formación del juicio de los ciudadanos, quienes al reunirse en la polis evalúan acciones concretas, otorgando así a la afectividad un importante rol en la conformación de las comunidades y la vida en común (cf. CÁRDENAS, 2011). Aristóteles introduce la retórica afectiva, es decir la vinculación que la palabra tiene con las pasiones, vinculación del logos con el pathos. Bruno, en este sentido, escribe también una reflexión sobre las relaciones civiles

4 Presentamos esta tesis en un sentido parcial porque es posible hacer otras lecturas menos materialistas al respecto, en especial si se toman en cuenta las afirmaciones de Bruno sobre el amor en Los heroicos furores, donde distingue con especial énfasis los estadios del amor en la medida en que se va despojando de los nudos materiales hasta llegar al amor divino. 
desde la perspectiva de los vínculos en un sentido similar al de Aristóteles, pero parece que Bruno enmarca las relaciones civiles dentro de una especie de "comunidad cósmica" en donde los vínculos regulan el trato de los hombres no solamente entre ellos sino con los otros seres del mundo.

Si relacionamos, como lo hace Bruno, los vínculos y los afectos, tendríamos que tener presente que en primer lugar los vínculos son afectos que no se oponen ni han de ser conducidas por la razón, pues cada vínculo es una racionalidad, "se conduce a los vínculos a destino a través del conocimiento en general" (BRUNO, 2007, p.84), y a su vez tampoco se separan de lo práctico, porque vincular es primeramente un hacer; en este contexto los vínculos brunianos ligan como un hacer-querer-pensar; hay aquí una crítica explícita al mundo griego en este sentido:

los griegos no reconducían al conocimiento racional, sino a la fortuna, el hecho de que alguien fuese vinculado por amor, odio u otro sentimiento....para nosotros, el amor, como cualquier otro sentimiento es una forma muy efectiva de conocer; es más bien el proceder discursivo racional y argumentativo por el cual sobre todo los hombres se dejan vincular y que no se coloca en absoluto entre las formas primarias de conocimiento...sin conocimiento y pasión nadie tiene la posibilidad de ligar (BRUNO, 2007, p.97s).

Por otra parte el rol de la imaginación en los vínculos es crucial, a tal punto que

la imaginación sin verdad puede vincular verdaderamente...suponiendo que no exista el infierno, la creencia imaginaria en el infierno, sin fundamento verdadero, produce verdaderamente un infierno: la imagen fantástica tiene su verdad, con la consecuencia de que ella opera realmente, $y$ real y potentemente queda sujetado aquel que se deja vincular (BRUNO, 2007, p.99).

Justamente porque los vínculos son un hacer, el rol de la imaginación adquiere el poder de dar realidad.

Varias de estas cuestiones están siendo replanteadas de forma relevante a partir del sXX: el cuestionamiento de la pasividad de las pasiones, la unidad de pensamiento y afectividad, el rol político de la afectividad en la conformación de la comunidad, su carácter performativo y su relación con la imaginación, entre otras muchas cuestiones. Humberto Giannini ha trabajado cuidadosamente la cuestión de los vínculos a los otros, afirmando que hay algo que va más allá del lenguaje y es la comunicación, que es un acto de comunicar. Ahora bien, la cuestión esencial aquí, para Giannini, gran conocedor de Bruno es ¿qué comunicamos cuando comunicamos? y justamente lo que comunicamos son los vínculos a los otros. "Lo que se comunica en la comunicación es el vínculo al otro: comunicamos vínculos. Toda la vida humana consiste en acercarnos al otro para comunicarles algo que es el vínculo que queremos tener con esa persona" (1996). 
Hemos intentado mostrar aquí que la filosofía de Giordano Bruno desde esta perspectiva puede realizar aportes importantes y aquí hemos querido hacer una primera demarcación de líneas que podrían seguir siendo trabajadas en función de repensar otra vez nuestra comunidad.

Valentina Bulo es doctora en Filosofía por la Universidad Complutense de Madrid y actualmente académica de Istituto de Estudios Avanzados de la Universidad de Santiago; ha publicado diversos artículos sobre la temática del cuerpo y la afectividad y los libros El temblor del ser:cuerpo y afectividad en el pensar tardío de Heidegger (Biblos, 2012) y Tonos de realidad: pensar la afectividad en la filosofía de Xavier Zubiri (RIL, 2013) .

valentina.bulo@usach.cl

\section{Referencias}

ARISTÓTELES. Retórica. Madrid: Centro de estudios Políticos y Constitucionales, 1999.

BRUNO, Giordano. De la Causa, Principio y Uno. Buenos Aires: Editorial Losada, 1941.

De la magia, de los vínculos en general. Buenos Aires: Ed. Cactus, 2007.

Cábala del Caballo Pegaso. Madrid: Alianza Editorial, 1990.

Expulsión de la bestia triunfante. Madrid: Alianza Editorial, 1995.

Los Heroicos Furores. Madrid: Editorial Tecnos, 1987.

Sobre el infinito universo y los mundos. Madrid: Alianza Editorial, 1998.

CÁRDENAS, Luz Gloria. Aristóteles: Retórica, pasiones y persuasión. Bogotá: Ed. San Pablo, 2011.

CASTRO, Antonio. Giordano Bruno. Madrid: Ediciones del Orto, 2000.

FICINO, Marcilio. De amore, comentario a "El Banquete" de Platón. Madrid: Tecnos, 1986.

GIANNINI, Humberto. "Heroicos furores". Disponible en http://www.geocities.ws/fdomauricio/ bruno2.htm, Acceso el 24 de enero de 2016.

Discurso Premio Jorge Millas U. Austral de Chile, en https://mega.co.nz/\#!BQRThbbJ\%21xt NiOJNsOdvzOel-st6MJNOreBUUB54uzcylkzsOl1M. Acceso el 24 de enero de 2016.

GÓMEZ DE LIAÑO, Ignacio. Mundo, Magia y Memoria. Madrid: Editorial Taurus, 1973.

GONZÁLEZ, María Rosario. "Introducción" en Heroicos Furores. Madrid: Editorial Tecnos, 1987.

GRANADA, Miguel Ángel. La reivindicación de la filosofía en Giordano Bruno. Barcelona: Herder, 2005.

HEIDEGGER, Martin. Ser y tiempo. Santiago: Ed. Universitaria, 1997.

MOLINA, Eduardo. Lucrecio: textos sobre Venus, el amor y la muerte. Onomazein 3: p. 241-256, 1998. 
MONDOLFO, Rodolfo. Tres filósofos del renacimiento. Buenos Aires: Editorial Losada, 1947.

SERRES, Michel. El nacimiento de la física en el texto de Lucrecio, caudales y turbulencias. Madrid: Ed. Pre-textos, 1994.

Texto recebido em março e aprovado em abril de 2015. 